\title{
Undecanoic Acid, Lauric Acid, and N-Tridecanoic Acid Inhibit Escherichia coli Persistence and Biofilm Formation
}

\author{
Xing Jin ${ }^{\dagger}$, Jiacheng Zhou ${ }^{\dagger}$, Gabriella Richey, Mengya Wang, Sung Min Choi Hong, and \\ Seok Hoon Hong*
}

Department of Chemical and Biological Engineering, Illinois Institute of Technology, Chicago, IL 60616, USA

Persister cell formation and biofilms of pathogens are extensively involved in the development of chronic infectious diseases. Eradicating persister cells is challenging, owing to their tolerance to conventional antibiotics, which cannot kill cells in a metabolically dormant state. A high frequency of persisters in biofilms makes inactivating biofilm cells more difficult, because the biofilm matrix inhibits antibiotic penetration. Fatty acids may be promising candidates as antipersister or antibiofilm agents, because some fatty acids exhibit antimicrobial effects. We previously reported that fatty acid ethyl esters effectively inhibit Escherichia coli persister formation by regulating an antitoxin. In this study, we screened a fatty acid library consisting of 65 different fatty acid molecules for altered persister formation. We found that undecanoic acid, lauric acid, and N-tridecanoic acid inhibited E. coli BW25113 persister cell formation by 25-, 58-, and 44-fold, respectively. Similarly, these fatty acids repressed persisters of enterohemorrhagic E. coli EDL933. These fatty acids were all medium-chain saturated forms. Furthermore, the fatty acids repressed Enterohemorrhagic $E$. coli (EHEC) biofilm formation (for example, by 8-fold for lauric acid) without having antimicrobial activity. This study demonstrates that medium-chain saturated fatty acids can serve as antipersister and antibiofilm agents that may be applied to treat bacterial infections.

Keywords: E. coli, persister cells, biofilms, medium-chain saturated fatty acids

Received: August 15, 2020 Accepted: October 12, 2020

First published online: October 13, 2020

* Corresponding author Phone: +1-312-567-8950 E-mail: shong26@iit.edu

${ }^{\dagger}$ These authors contributed equally to this study.

Supplementary data for this paper are available on-line only at http://jmb.or.kr.

pISSN 1017-7825 eISSN 1738-8872

Copyright(C) 2021 by The Korean Society for Microbiology and Biotechnology

\section{Introduction}

Persister cells are tolerant to conventional antibiotic treatment because they are metabolically dormant or grow slowly without acquiring inherent antibiotic resistance via genetic modifications [30], whereas resistant cells undergo genetic changes that block antibiotic activity [16]. Persister pathogens become active after the level of antibiotics decreases and thus cause chronic recalcitrant infections [19]. Persister cells are also highly tolerant to environmental stresses, such as low pH [25], nutrient starvation [4], hyperosmolarity [37], or heat shock [5]. Because of their high tolerance, persister cells are enriched in biofilms [21], sessile multi-microbial communities that are formed in response to environmental stresses [3] through secreted self-synthesized polymeric matrices [17]. Many bacteria, including pathogens, can form biofilms, but inhibiting biofilms is challenging because of poor antibiotic penetration, biofilm-specific gene regulation, and persister cell formation [35]. Hence, identifying new antimicrobial agents other than antibiotics is critical to decrease persister cell formation.

Fatty acid molecules, composed of a chain of carbon atoms with attached hydrogen atoms, are widely used in therapeutics, food preservation, and agriculture [12]. Some fatty acids inhibit or kill bacteria directly [51], whereas others affect virulence factors or prevent bacterial adhesion [44]. Fatty acids are produced in natural sources, such as plants that are inexpensive and non-toxic [43]. The antimicrobial activities of fatty acids depend on the structure, chain length, and degree of saturation [36]. As a result of these properties, fatty acids are considered as an alternative to conventional antibiotics for the treatment of infectious diseases. Furthermore, fatty acids exhibit antibiofilm properties in relation to antivirulence [28]. However, the roles of fatty acid molecules in altering persister cells have not been extensively studied. cis-2-decenoic acid produced from Pseudomonas aeruginosa was first identified for decreasing bacterial cells in a dormant state by causing them to transition to a metabolically active state via enhancing the expression of metabolic marker transcripts [32]. This compound has also been found to inhibit biofilm formation through enhancing biofilm dispersion [31]. Recently, we reported that ethyl esters of unsaturated fatty acids, including ethyl trans-2-decenoate, ethyl trans-2-octenoate, and ethyl cis-4-decenoate, repress Escherichia coli persister formation by regulating the antitoxin HipB [47]. Therefore, fatty acid molecules can be applied to control persister cell formation, thus preventing bacterial infections.

In this study, we screened 65 fatty acids with chain lengths of 10 to 24 carbons, including saturated and unsaturated forms, to determine whether combined antibiotic and fatty acid treatment might decrease persister 
Table 1. List of three selected fatty acids.

Undecanoic acid

Undecanoic acid, lauric acid, and N-tridecanoic acid were selected as antipersister molecules from BW25113 persister cell screening.

cell formation. We identified antipersister and/or antibiofilm fatty acid molecules with the potential to be used with antibiotics to treat bacterial infections efficiently.

\section{Materials and Methods}

Bacterial Strains and Fatty Acids

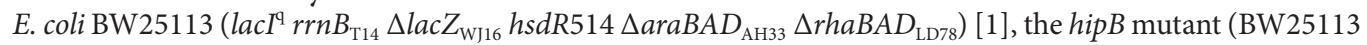
$\triangle$ hipB) of the same strain, and enterohemorrhagic E. coli EDL933 (ATCC 43895) were used in this study. E. coli strains were cultured in $3 \mathrm{ml}$ of LB medium at $220 \mathrm{rpm}$ at $37^{\circ} \mathrm{C}$ overnight. The SCREEN-WELL Fatty Acid Library, purchased from Enzo Life Science (USA), contained 65 fatty acids (Table S1) dissolved in dimethyl sulfoxide (DMSO) with a stock concentration of $10 \mathrm{mM}$. Undecanoic acid (AC173970250), lauric acid (AC167281000), and $\mathrm{N}$-tridecanoic acid (AC139520100) were purchased from Acros Organics (Morris Plains, USA) and are listed in Table 1.

\section{Persister Cell Formation Assays}

Persister assays were conducted according to previously reported procedures [24]. Briefly, overnight E. coli cultures were diluted 100 -fold in $25 \mathrm{ml}$ of fresh LB medium in sterilized $250 \mathrm{ml}$ flasks and incubated at $220 \mathrm{rpm}$ at $37^{\circ} \mathrm{C}$ until an $\mathrm{OD}_{600 \mathrm{~nm}} 0.7-0.9$ was reached. Late stationary phase cells were harvested after overnight growth $(18 \mathrm{~h}$ incubation following inoculation). Exponential phase or late stationary phase cells were harvested by centrifugation at $12,000 \times g$ for $5 \mathrm{~min}$, washed twice with $0.85 \% \mathrm{NaCl}$ solution, and adjusted to $\mathrm{OD}_{600 \mathrm{~nm}} 1.0$ with fresh LB. Then, the cells were exposed to each fatty acid at a final concentration of $0.1 \mathrm{or} 1 \mathrm{mM}$ in the presence of $5 \mu \mathrm{g} / \mathrm{ml}$ ciprofloxacin, $100 \mu \mathrm{g} / \mathrm{ml}$ ampicillin, or $100 \mu \mathrm{g} / \mathrm{ml}$ kanamycin for $3 \mathrm{~h}, 6 \mathrm{~h}$, or $24 \mathrm{~h}$ at $220 \mathrm{rpm}$ at $37^{\circ} \mathrm{C}$. Cells were pelleted, washed twice with $0.85 \% \mathrm{NaCl}$ solution, and diluted serially $\left(10^{1}-10^{4}\right.$ dilution). Then $10 \mu \mathrm{l}$ was applied to LB agar plates, which were incubated at $37^{\circ} \mathrm{C}$. Persister cells were quantified by CFU counting.

\section{Biofilm Formation Assays}

Biofilm formation assays were conducted according to a previously described method [15] with modifications. Briefly, overnight cultures were adjusted to an $\mathrm{OD}_{600 \mathrm{~nm}}$ of 0.01 (approximately $5 \times 10^{6} \mathrm{CFU} / \mathrm{ml}$ ) with LB medium, and then $1.5 \mathrm{ml}$ of adjusted culture was added to a polypropylene culture tube (Falcon PN352006, Corning, USA) with or without fatty acids. After incubation at $37^{\circ} \mathrm{C}$ for $24 \mathrm{~h}$ without shaking, the planktonic cultures were discarded, and the culture tubes containing biofilm were rinsed three times with $3 \mathrm{ml}$ of $0.85 \% \mathrm{NaCl}$ solution. Biofilm cells were carefully collected with a cotton swab [42] and resuspended in $3 \mathrm{ml}$ of $0.85 \% \mathrm{NaCl}$ solution via vortexing for $30 \mathrm{~s}$. Biofilm cells were diluted serially $\left(10^{1}-10^{6}\right.$ dilution), and $10 \mu \mathrm{l}$ was applied on LB agar plates, which were incubated at $37^{\circ} \mathrm{C}$. Biofilm cells were quantified as colony forming units $(\mathrm{CFU})$ per surface area $\left(\mathrm{cm}^{2}\right)$.

\section{Growth Measurement}

Overnight cultures were adjusted to $\mathrm{OD}_{600 \mathrm{~nm}} 0.05$ with fresh $\mathrm{LB}$ medium, and then $200 \mu \mathrm{l}$ cell suspensions were added to a 96 -well plate. After the addition of different concentrations of fatty acids, cell growth was measured at $\mathrm{OD}_{600 \mathrm{~nm}}$ every $20 \mathrm{~min}$ at $37^{\circ} \mathrm{C}$ on a Synergy HTX plate reader in fast shaking mode (Biotek, USA). Each data point was obtained from three replicate wells with two independent cultures.

\section{Statistical Analysis}

Statistical analysis was performed with two-tailed Student's $t$-test between ciprofloxacin only or DMSO control and each fatty acid treatment. The statistical significance is indicated in the figure with ${ }^{\star}(p<0.001)$. All reported data are average \pm standard deviation.

\section{Results and Discussion}

Identification of Undecanoic Acid, Lauric Acid, and N-Tridecanoic Acid as Inhibitors of E. coli BW25113 Persister Cell Formation

To explore whether fatty acids can inhibit persister cell formation, we examined the ability of 65 fatty acids to alter persister cell formation. These fatty acids ranged in length from 10 to 24 carbons and were saturated or unsaturated. First, we treated exponential phase E. coli BW25113 cells with $5 \mu \mathrm{g} / \mathrm{ml}$ of ciprofloxacin and $0.1 \mathrm{mM}$ of 
A

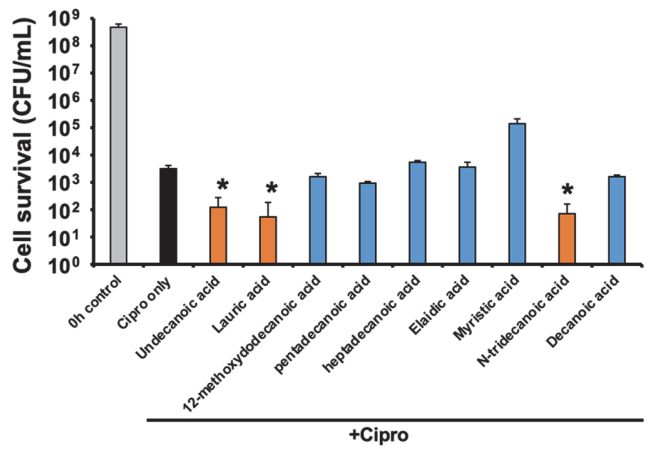

B

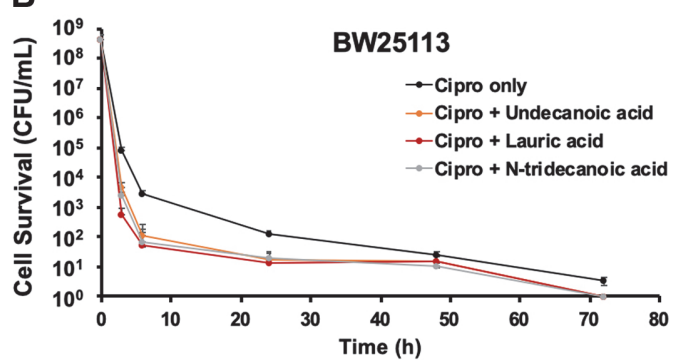

C

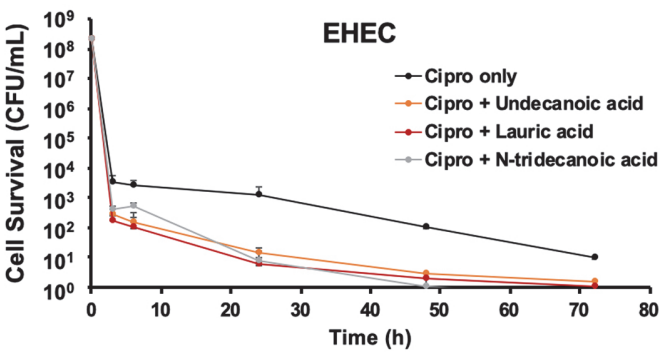

Fig. 1. Fatty acid induced alterations in E. coli persister formation. (A) Exponentially grown E. coli BW25113 cells were exposed to $5 \mu \mathrm{g} / \mathrm{ml}$ of ciprofloxacin (Cipro) and $1 \mathrm{mM}$ of nine fatty acids for $6 \mathrm{~h}$ in LB medium. (B) Exponentially grown BW25113 and (C) EDL933 (Enterohemorrhagic E. coli) cells were exposed to $5 \mu \mathrm{g} / \mathrm{ml}$ of ciprofloxacin and $1 \mathrm{mM}$ of undecanoic acid, lauric acid, and N-tridecanoic acid for 3, 6, 24, 48, and $72 \mathrm{~h}$ in LB medium. Error bars indicate the standard deviation of two independent cultures with three replicates. ${ }^{*}$ Indicates a significant difference relative to ciprofloxacin treatment only, with a $p$-value $<0.001$.

each fatty acid for $6 \mathrm{~h}$ at $37^{\circ} \mathrm{C}$ at $220 \mathrm{rpm}$ and compared the levels of persister cells to those after ciprofloxacin only treatment. This screen resulted in the selection of nine fatty acids that decreased persister cells more than twofold. We then performed a second persister experiment with the nine candidate fatty acids by increasing their concentration to $1 \mathrm{mM}$ to maximize their persister inhibition effect under the same incubation conditions (Fig. 1A). Undecanoic acid, lauric acid, and $\mathrm{N}$-tridecanoic acid were identified as persister inhibitors that consistently decreased persister cell formation in the BW25113 strain, by 25-, 58-, and 44-fold, respectively. (Fig. 1A). Next, we examined persister cell formation at different time points. The incubation time was increased up to $72 \mathrm{~h}$, and a biphasic cell survival curve [2] was obtained after ciprofloxacin treatment (Fig. 1B). Cotreatment with ciprofloxacin and each fatty acid further decreased the levels of persisters over the examination period, to levels below those after ciprofloxacin-only treatment. After longer time incubation, $(24,48$, and $72 \mathrm{~h})$, the cell survival after cotreatment was almost negligible (Fig. 1B). These results indicate that undecanoic acid, lauric acid, and $\mathrm{N}$-tridecanoic acid repress persister formation in the BW25113 strain.

Interestingly, the three fatty acids obtained from the persister inhibition screening were all saturated fatty acids of medium-chain length (undecanoic acid, 11 carbon atoms (C11); lauric acid, C12; and N-tridecanoic acid, C13) (Table 1), whereas a collection of various-length (C10 to C24) fatty acids of saturated and unsaturated states were studied (Table S1). cis-2-decenoic acid, which is a known as antipersister and antibiofilm fatty acid, is mediumchain (C10) and unsaturated [32]. Previously, we investigated nine fatty acids and their derivatives [47] that were unsaturated and structurally similar to cis-2-decenoic acid and found that medium-chain fatty acid derivatives containing ethyl esters (ethyl trans-2-decenoate, ethyl trans-2-octenoate, and ethyl cis-4-decenoate) inhibit persister formation [47]. Longer-chain fatty acids with greater than C14, such as pentadecanoic acid, heptadecanoic acid, eliadic acid, and myristic acid (Fig. 1A), shorter-chain fatty acid such as decanoic acid (Fig. 1A), and the fatty acid derivatives with C6 to C9 [47] were not able to decrease persister level. Although cellular responses depend on specific fatty acid structural characters, including length, saturation level, and type and location of functional groups [33], nonetheless the chain length of fatty acids may be a critical consideration with respect to antipersister activity, according to previous [32, 47] and current studies. Regulation of bacterial toxin-antitoxin systems [47], increasing protein synthesis [32], or influencing membrane fluidity [28] by fatty acids might cause cells to be more susceptible to antibiotic treatment. However, undecanoic acid, lauric acid, and $\mathrm{N}$-tridecanoic acid in this study were all saturated forms distinct from the previously reported antipersister fatty acid compounds, thus suggesting that they may have a different mechanism of inhibiting bacterial persisters. Further systematic studies will be necessary to understand the specific interactions of medium-chain saturated fatty acids with antibiotics or bacteria during persister formation. 
A

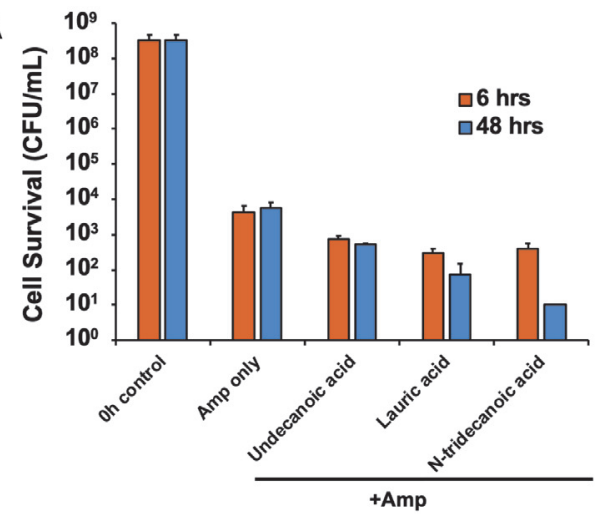

$B$

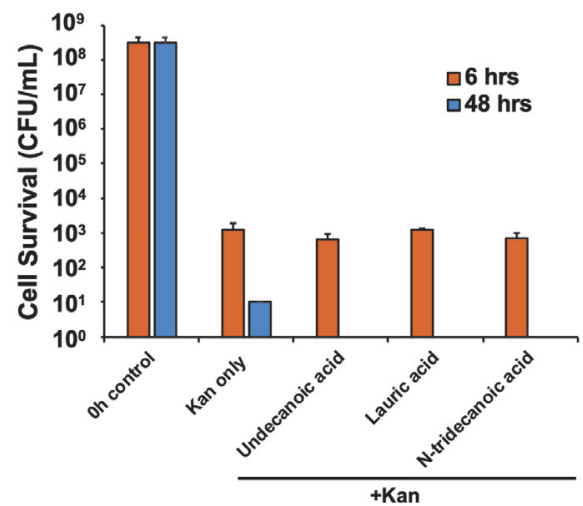

Fig. 2. EHEC persister cell formation in the presence of different antibiotics. EHEC persister formation with $100 \mu \mathrm{g} / \mathrm{ml}$ of (A) ampicillin (Amp) and (B) kanamycin (Kan) together with $1 \mathrm{mM}$ of fatty acids for 6 and $48 \mathrm{~h}$ in LB. Error bars indicate the standard deviation of two independent cultures with three replicates. ${ }^{*}$ Indicates a significant difference relative to Cipro treatment only, with a $p$-value $<0.001$.

\section{Undecanoic Acid, Lauric Acid, and N-Tridecanoic Acid Inhibit Enterohemorrhagic E. coli (EHEC) Persister Formation}

To investigate the effects of these fatty acids on other E. coli strains, we examined the time course of persister formation of the EHEC strain, a pathogenic E. coli responsible for outbreaks of bloody diarrhea and hemolytic uremic syndrome, and a major source of food poisoning [38]. The persister level of EHEC in the presence of ciprofloxacin $5 \mu \mathrm{g} / \mathrm{ml}$ was $0.0005 \%\left(2.5 \times 10^{3} \mathrm{CFU} / \mathrm{ml}\right)$ after a $3 \mathrm{~h}$ incubation (Fig. $1 \mathrm{C}$ ), a value 20 -fold lower than that of BW25113 (Fig. 1B). This result was consistent with our previous observation of EHEC persister cell formation [47]. When we added $1 \mathrm{mM}$ of undecanoic acid, lauric acid, and $\mathrm{N}$-tridecanoic acid during EHEC persister formation for $3 \mathrm{~h}$, the persister level was 11-, 21-, and 9-fold lower, respectively, than that after ciprofloxacin-only treatment (Fig. 1C). Moreover, extended incubation (up to $72 \mathrm{~h}$ ) with each fatty acid and ciprofloxacin resulted in nearly complete inactivation of EHEC cells (Fig. 1C). Therefore, undecanoic acid, lauric acid, and N-tridecanoic acid effectively inhibit persister formation of E. coli.

Typically, antibiotics repress bacterial growth through different inhibition mechanisms. For example, ciprofloxacin (fluoroquinolone) inhibits DNA replication and repair, ampicillin ( $\beta$-lactam) blocks cell-wall synthesis, and kanamycin (aminoglycosides) represses protein synthesis [14]. Therefore, we investigated the effects of the mediumchain saturated fatty acids during EHEC persister formation in the presence of different classes of antibiotics in addition to ciprofloxacin. Specifically, we examined EHEC persister formation with $100 \mu \mathrm{g} / \mathrm{ml}$ of ampicillin (Fig. 2A) or kanamycin (Fig. 2B) in the presence of $1 \mathrm{mM}$ of each fatty acid for 6 and 48 h. Similarly to the results with ciprofloxacin and each fatty acid cotreatment (Fig. 1B), the addition of undecanoic acid, lauric acid, and Ntridecanoic acid repressed persister cell formation caused by ampicillin treatment (6-, 14-, and 10-fold lower, respectively, than that after ampicillin-only treatment after 6 h; Fig. $2 \mathrm{~A})$. However, we did not observe a noticeable difference during kanamycin treatment with or without fatty acids (Fig. 2B). Cell survival after $48 \mathrm{~h}$ of kanamycin exposure $(10 \mathrm{CFU} / \mathrm{ml})$ was considered as negligible as it was close to the complete cell killing with the cotreatment (Fig. 2B). These results suggest that the fatty acid molecules that we tested behave differently when persister cells are formed through various bacterial inactivation mechanisms in the presence of different antibiotics.

Persister cell formation is known to be an antibiotic-specific response rather than a global metabolic dormancy [20]. The SOS response after DNA damage by ciprofloxacin induces toxin-antitoxin (TA) transcripts in E. coli, thus resulting in persister formation [50], and ampicillin treatment induces TA pairs, thereby increasing persister cells [40]. However, motility and amino acid biosynthesis play important roles in gentamycin (aminoglycoside)induced persister cell formation [41]. We previously reported that unsaturated fatty acid ethyl esters with ciprofloxacin increase the antitoxin HipB, thus decreasing persister formation, and similar persister inhibition was achieved after cotreatment with ampicillin [47]. We performed persister assay of E. coli BW25113 $\Delta$ hipB strain in the presence of ciprofloxacin and each fatty acid. Corroborating to the previous study [47], the persister level of hipB mutant upon ciprofloxacin was 100-fold higher (Fig. S1) that that of the wild-type BW25113 strain (Fig. 1B). Persister formation of $h i p B$ mutant was decreased by the cotreatment of fatty acid and ciprofloxacin in comparison to the ciprofloxacin-only treatment after 6 and $48 \mathrm{~h}$ incubation (Fig. S1), which performed similar trend to the persister formation of wild-type BW25113 strain by the cotreatment (Fig. 1B). Hence, the decrease of persister cell formation by undecanoic acid, lauric acid, and $\mathrm{N}$-tridecanoic acid was not associated with the work of antitoxin HipB. However, because E. coli possesses multiple TA systems [45], the possibility remains that undecanoic acid, lauric acid, and N-tridecanoic acid might influence TA-related cellular regulation and consequently could enhance persister cell control.

We determined that fatty acids did not repress persister formation induced by kanamycin (Fig. 2B). Kanamycin binds the $30 \mathrm{~S}$ ribosomal subunit and inhibits protein synthesis [6]; as a result, ribosome dimerization induced by the presence of aminoglycosides, including kanamycin, results in the ribosome hibernation and thereby increases bacterial tolerance [34]. Recently, the alarmone guanosine pentaphosphate/tetraphosphate (ppGpp) ribosome 
dimerization persister model has been proposed to understand how cells enter and exit the persister state [46, 48]. Persister cells are directly formed by inactivating ribosomes via ppGpp with the evidence that persister cells contain a large fraction of $100 \mathrm{~S}$ ribosomes [46]. Therefore, it is possible that the fatty acids might interact with ribosomes to interrupt the pathway of persistence formation, whereas kanamycin-induced persister cells containing inactivated ribosomes might not be affected by the fatty acids.

\section{Persister Formation in Stationary Phase Cells Is Not Affected by Fatty Acids}

To investigate the effect of fatty acids on stationary phase persister cell formation, we directly harvested the overnight culture (18 h after inoculation) and adjusted its optical density to 1.0 with fresh LB media in the presence of $1 \mathrm{mM}$ of each fatty acid and $5 \mu \mathrm{g} / \mathrm{ml}$ of ciprofloxacin. The population of persister cells in the stationary phase with ciprofloxacin-only treatment was increased by more than 10-fold in comparison to that in the exponential phase. However, unlike the exponential phase of persister cell formation, the effect of fatty acid inhibition on persister cell formation was diminished in the stationary phase cells of BW25113 and EHEC (Fig. S2). Stationary phase cells have more robust membranes with a rigid cell envelope, a highly cross-linked cell wall, and a reduced membrane fluidity in comparison to exponential phase cells, which exhibit a high membrane fluidity [22]. Bacteria can incorporate free exogenous fatty acids from its environment to their cellular membrane [13], such that the incorporation of fatty acids into the membrane may increase membrane fluidity and thereby enhance antimicrobial susceptibility $[11,28]$. Our results that fatty acids are effective toward decreasing the persister formation of exponential phase cells but are less effective in regard to stationary phase cells, implying that undecanoic acid, lauric acid, and $\mathrm{N}$-tridecanoic acid might increase membrane fluidity and improve the ciprofloxacin efficacy for inhibiting persister formation. It remains necessary to elucidate the relation between the cell membrane fluidity, persister formation, and the effect of fatty acids on membrane fluidity.

\section{Undecanoic Acid, Lauric Acid, and N-Tridecanoic Acid Do Not Exhibit Antimicrobial Activity against EHEC Cells}

Some fatty acids, such as linolenic acid, myristic acid, and lauric acid, have antimicrobial activity against microorganisms such as Bacillus subtilis, Listeria monocytogenes, Mycobacterium smegmatis, and Staphylococcus aureus [7]. Because the identified fatty acids (undecanoic acid, lauric acid, and N-tridecanoic acid) effectively inhibited persister cell formation (Fig. 1), we investigated whether their inhibition effects might be derived from their antimicrobial activity. Specifically, we monitored the growth of EHEC cells in the presence of $1 \mathrm{mM}$ of undecanoic acid, lauric acid, or N-tridecanoic acid (Fig. 3). The final cell growth OD in the late stationary growth phase after $15 \mathrm{~h}$ was similar among the DMSO control and fatty acid-treated samples with no significant difference (Fig. 3), indicating that undecanoic acid, lauric acid, and $\mathrm{N}$-tridecanoic acid do not exhibit antimicrobial activity against EHEC cells. However, there was a slight improvement in cell growth with fatty acids, particularly during 3 to $13 \mathrm{~h}$ of incubation (Fig. 3). Reportedly, many fatty acids exhibit antimicrobial activity in a selective manner [7, $28,51]$. On the one hand, fatty acids inhibit Gram-positive bacteria $[7,10,12]$. On the other hand, fatty acids are metabolized in bacteria to maintain lipid homeostasis as well as for use as carbon and energy sources [18, 23]. Therefore, EHEC cells might utilize fatty acids to enhance cellular growth during the exponential and early stationary growth phases. Previous studies also suggest that the antipersister activity of fatty acid molecules is not derived from the antimicrobial activity but is based on specific intracellular interactions affecting protein synthesis or regulation [32, 47]. Fatty acid molecules may not induce bacterial burden (Fig. 3) but may synergistically increase the efficacy of antibiotics in killing bacteria (Fig. 1). Persister cell inhibition without direct antimicrobial activity might be an attractive approach, because such antipersister compounds may mitigate the development of antimicrobial resistance of bacteria by maximizing the function of conventional antibiotics in inactivating bacteria.

\section{Fatty Acids Repress EHEC Biofilm Formation}

High persister populations in biofilms enhance the tolerance of biofilm cells $[8,39]$. Because the examined fatty acid molecules inhibited persister cell formation, we reasoned that these fatty acids might be applied for repressing

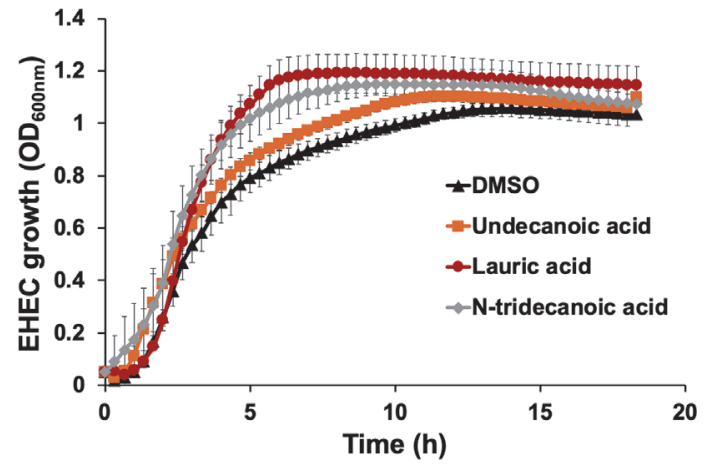

Fig. 3. EHEC growth with undecanoic acid, lauric acid, and N-tridecanoic acid. The growth of EHEC culture was monitored until $18 \mathrm{~h}$ in the presence of $1 \mathrm{mM}$ of each fatty acid at $37^{\circ} \mathrm{C}$ in $\mathrm{LB}$. Cell growth at $\mathrm{OD}_{600 \mathrm{~mm}}$ was measured every $20 \mathrm{~min}$. Error bars indicate standard deviations of two independent cultures with three replicates. 


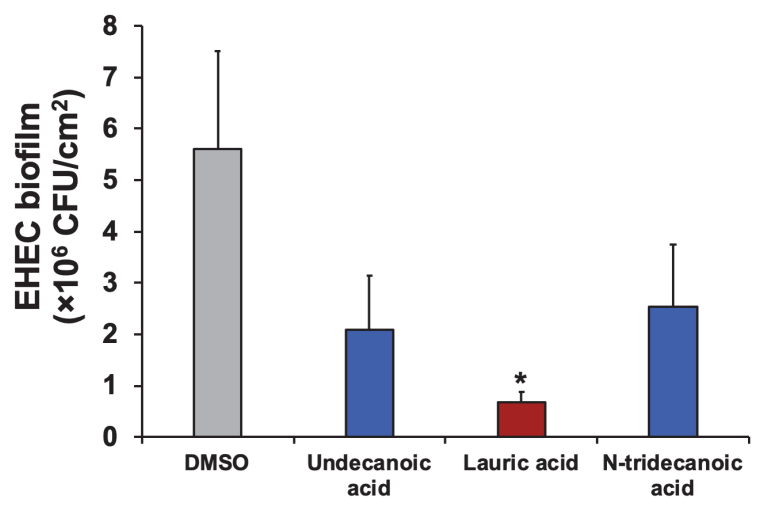

Fig. 4. EHEC biofilm formation. EHEC biofilms were formed in the presence of $1 \mathrm{mM}$ of undecanoic acid, lauric acid, and $\mathrm{N}$-tridecanoic acid for $24 \mathrm{~h}$ at $37^{\circ} \mathrm{C}$ without shaking. Error bars indicate standard deviations of two independent cultures with three replicates. ${ }^{*}$ Indicates a significant difference relative to the DMSO control, with a $p$-value $<0.001$.

biofilm formation. We exposed EHEC cells to undecanoic acid, lauric acid, and N-tridecanoic acid during $24 \mathrm{~h}$ of biofilm formation. As expected, all fatty acids tested repressed EHEC biofilms, and lauric acid exhibited the highest efficacy (8-fold; Fig. 4). Lauric acid inhibits Clostridium difficile biofilm formation and disrupts preformed biofilms through the antimicrobial activity of lauric acid [49]. Recently, we reported that the combined treatment of lauric acid and the antimicrobial peptide nisin represses the development of nisin resistance of $L$. monocytogenes and inactivates cells in biofilms [52]. However, our results indicated that without having antimicrobial activity toward EHEC (Fig. 3), lauric acid inhibits EHEC biofilm formation (Fig. 4). Fatty acids can serve as signaling molecules in cell-cell communication, e.g., diffusible signal factor [27]. A previous study has indicated that cis-2-decenoic acid functions as a signaling molecule that stimulates the biofilm dispersion response and thus inhibits biofilm formation by Gram-positive and Gram-negative bacteria [9, 31]. However, these fatty acid signaling molecules are typically short-chain unsaturated fatty acids with carbon 2 in a cis configuration [31]. Fatty acids also can regulate virulence factors and prevent bacterial adhesion [9]. For example, some saturated fatty acids, such as lauric acid (12 carbon atoms), myristic acid (14 carbon atoms), and palmitic acid (16 carbon atoms), inhibit Proteus mirabilis biofilm formation by regulating swarming and virulence factor expression [29]. Various fatty acids possess ability to inhibit biofilm development by affecting the adhering surface and cell membrane fluidity [28]. For example, linoleic acid inhibits $P$. aeruginosa biofilms by increasing the membrane permeability of the bacteria [26]. Given the close relationships observed among persister cells, biofilms, and virulence factors, as well as our observations that undecanoic acid, lauric acid, and N-tridecanoic acid exhibited antipersister and antibiofilm activities, medium-chain saturated fatty acids may play important roles by connecting persister cell repression, biofilm inhibition, and potentially virulence regulation.

This study demonstrates that the three fatty acid molecules undecanoic acid, lauric acid, and N-tridecanoic acid effectively inhibit $E$. coli persistence as well as biofilm formation. Fatty acids are non-toxic, naturally produced products that are safe to humans [43] and have promise for applications in therapeutics, food preservation, and agriculture [12]. Therefore, medium-chain saturated fatty acids may serve as new antipersister and/or antibiofilm agents to combat antibiotic-resistant bacterial infections.

\section{Acknowledgments}

This study was supported by Illinois Institute of Technology, the Research Experiences for Undergraduates (REU) program of the National Science Foundation (1757989), and the National Institute of Allergy and Infectious Diseases of the National Institutes of Health (R15AI130988).

\section{Conflict of Interest}

The authors have no financial conflicts of interest to declare.

\section{References}

1. Baba T, Ara T, Hasegawa M, Takai Y, Okumura Y, Baba M, et al. 2006. Construction of Escherichia coli K-12 in-frame, single-gene knockout mutants: the Keio collection. Mol. Syst. Biol. 2: 2006.0008.

2. Balaban NQ, Helaine S, Lewis K, Ackermann M, Aldridge B, Andersson DI, et al. 2019. Definitions and guidelines for research on antibiotic persistence. Nat. Rev. Microbiol. 17: 441-448.

3. Balcázar JL, Subirats J, Borrego CM. 2015. The role of biofilms as environmental reservoirs of antibiotic resistance. Front. Microbiol. 6: 1216 .

4. Brown DR. 2019. Nitrogen starvation induces persister cell formation in Escherichia coli. J. Bacteriol. 201: e00622-18.

5. Bruhn-Olszewska B, Szczepaniak P, Matuszewska E, Kuczyńska-Wiśnik D, Stojowska-Swędrzyńska K, Moruno Algara M. et al. 2018. Physiologically distinct subpopulations formed in Escherichia coli cultures in response to heat shock. Microbiol. Res. 209: 33-42.

6. Chulluncuy R, Espiche C, Nakamoto J, Fabbretti A, Milón P. 2016. Conformational response of 30S-bound IF3 to A-site binders streptomycin and kanamycin. Antibiotics. 5: 38.

7. Churchward CP, Alany RG, Snyder LAS. 2018. Alternative antimicrobials: the properties of fatty acids and monoglycerides. Crit. Rev. Microbiol. 44: 561-570. 
8. Ciofu O, Tolker-Nielsen T. 2019. Tolerance and resistance of Pseudomonas aeruginosa biofilms to antimicrobial agents-how P. aeruginosa can escape antibiotics. Front. Microbiol. 10: 913.

9. Davies DG, Marques CNH. 2009. A fatty acid messenger is responsible for inducing dispersion in microbial biofilms. J. Bacteriol. 191: 1393-1403.

10. Dayrit FM. 2015. The properties of lauric acid and their significance in coconut oil. J. Am. Oil Chem. Soc. 92: 1-15.

11. Delcour AH. 2009. Outer membrane permeability and antibiotic resistance. Biochim. Biophys. Acta - Proteins Proteomics. 1794: 808-816.

12. Desbois AP, Smith VJ. 2010. Antibacterial free fatty acids: activities, mechanisms of action and biotechnological potential. Appl. Microbiol. Biotechnol. 85: 1629-1642.

13. Dubois-Brissonnet F, Trotier E, Briandet R. 2016. The biofilm lifestyle involves an increase in bacterial membrane saturated fatty acids. Front. Microbiol. 7: 1673.

14. Fair RJ, Tor Y. 2014. Antibiotics and bacterial resistance in the 21st century. Perspect. Medicin. Chem. 6: 25-64.

15. Fang K, Jin X, Hong SH. 2018. Probiotic Escherichia coli inhibits biofilm formation of pathogenic E. coli via extracellular activity of DegP. Sci. Rep. 8: 4939.

16. Fisher RA, Gollan B, Helaine S. 2017. Persistent bacterial infections and persister cells. Nat. Rev. Microbiol. 15: $453-464$.

17. Flemming H-C, Wingender J, Szewzyk U, Steinberg P, Rice SA, Kjelleberg S. 2016. Biofilms: an emergent form of bacterial life. Nat. Rev. Microbiol. 14: 563-575.

18. Fujita Y, Matsuoka H, Hirooka K. 2007. Regulation of fatty acid metabolism in bacteria. Mol. Microbiol. 66: 829-839.

19. Gollan B, Grabe G, Michaux C, Helaine S. 2019. Bacterial persisters and infection: past, present, and progressing. Annu. Rev. Microbiol. 73: 359-385.

20. Goneau LW, Yeoh NS, MacDonald KW, Cadieux PA, Burton JP, Razvi H, et al. 2014. Selective target inactivation rather than global metabolic dormancy causes antibiotic tolerance in uropathogens. Antimicrob. Agents Chemother. 58: 2089-2097.

21. Hall CW, Mah T-F. 2017. Molecular mechanisms of biofilm-based antibiotic resistance and tolerance in pathogenic bacteria. FEMS Microbiol. Rev. 010: 276-301

22. Jaishankar J, Srivastava P. 2017. Molecular basis of stationary phase survival and applications. Front. Microbiol. 8: 2000.

23. Jimenez-Diaz L, Caballero A, Segura A. 2017. Pathways for the degradation of fatty acids in bacteria. pp. 1-23. In: Aerobic Utilization of Hydrocarbons, Oils and Lipids, Springer International Publishing.

24. Jin, X., Kightlinger, W., Kwon, Y.-C. and Hong, S. H. 2018. Rapid production and characterization of antimicrobial colicins using Escherichia coli-based cell-free protein synthesis. Synth. Biol. 3: ysy004.

25. Karki P, Mohiuddin SG, Kavousi P, Orman MA. 2020. Investigating the effects of osmolytes and environmental $\mathrm{pH}$ on bacterial persisters. Antimicrob. Agents Chemother. 64: e02393-19.

26. Kim HS, Ham SY, Jang Y, Sun PF, Park JH, Hoon Lee, .Park HD. 2019. Linoleic acid, a plant fatty acid, controls membrane biofouling via inhibition of biofilm formation. Fuel 253: 754-761.

27. Krzyżek P, Gościniak G. 2018. A proposed role for diffusible signal factors in the biofilm formation and morphological transformation of Helicobacter pylori. Turk. J. Gastroenterol. 29: 7-13.

28. Kumar, P., Lee, J. H., Beyenal, H. and Lee, J. 2020. Fatty acids as antibiofilm and antivirulence agents. Trends Microbiol. 28: $753-768$.

29. Liaw S-J, Lai H-C, Wang W-B. 2004. Modulation of swarming and virulence by fatty acids through the RsbA protein in Proteus mirabilis. Infect. Immun. 72: 6836-6845.

30. Maisonneuve E, Gerdes K. 2014. Molecular mechanisms underlying bacterial persisters. Cell 157: 539-548.

31. Marques CNH, Davies DG, Sauer K. 2015. Control of biofilms with the fatty acid signaling molecule cis-2-decenoic acid. Pharmaceuticals 8: 816-835.

32. Marques CNH, Morozov A, Planzos P, Zelaya HM. 2014. The fatty acid signaling molecule cis-2-decenoic acid increases metabolic activity and reverts persister cells to an antimicrobial-susceptible state. Appl. Environ. Microbiol. 80: 6976-6991.

33. McGaw LJ, Jäger AK, van Staden J. 2002. Antibacterial effects of fatty acids and related compounds from plants. South Afr. J. Bot. 68: 417-423.

34. McKay SL, Portnoy DA. 2015. Ribosome hibernation facilitates tolerance of stationary-phase bacteria to aminoglycosides. Antimicrob. Agents Chemother. 59: 6992-6999.

35. Olsen I. 2015. Biofilm-specific antibiotic tolerance and resistance. Eur. J. Clin. Microbiol. Infect. Dis. 34: 877-886

36. Petrovic S, Arsic A. 2016. Fatty acids: fatty acids. pp. 623-631. In: Encyclopedia of Food and Health, Elsevier Inc.

37. Poole K. 2012. Stress responses as determinants of antimicrobial resistance in Gram-negative bacteria. Trends Microbiol. 20: $227-234$.

38. Ranjbar R, Masoudimanesh M, Dehkordi FS, Jonaidi-Jafari N, Rahimi E. 2017. Shiga (Vero)-toxin producing Escherichia coli isolated from the hospital foods virulence factors, o-serogroups and antimicrobial resistance properties. Antimicrob. Resist. Infect. Control 6: 1-11.

39. Salisbury A-M, Woo K, Sarkar S, Schultz G, Malone M, Mayer DO. et al. 2018. Tolerance of biofilms to antimicrobials and significance to antibiotic resistance in wounds. Surg. Technol. Int. 33: 59-66.

40. Schuster CF, Mechler L, Nolle N, Krismer B, Zelder M-E, Götz F, et al. 2015. The MazEF toxin-antitoxin system alters the $\beta$-lactam susceptibility of Staphylococcus aureus. PLoS One 10: e0126118.

41. Shan Y, Lazinski D, Rowe S, Camilli A, Lewis K. 2015. Genetic basis of persister tolerance to aminoglycosides in Escherichia coli. MBio 6: e00078-15.

42. Shao X, Fang K, Medina D, Wan J, Lee JL, Hong SH. 2019. The probiotic, Leuconostoc mesenteroides, inhibits Listeria monocytogenes biofilm formation. J. Food Saf. 40: e12750.

43. Shilling M, Matt L, Rubin E, Visitacion MP, Haller NA, Grey SF, et al. 2013. Antimicrobial effects of virgin coconut oil and its medium-chain fatty acids on Clostridium difficile. J. Med. Food 16: 1079-1085.

44. Silva LN, Zimmer KR, Macedo AJ, Trentin DS. 2016. Plant natural products targeting bacterial virulence factors. Chem. Rev. 116: $9162-9236$.

45. Song S, Wood TK. 2020. A primary physiological role of toxin/antitoxin systems is phage inhibition. Front. Microbiol. $11: 1895$.

46. Song S, Wood TK. 2020. ppGpp ribosome dimerization model for bacterial persister formation and resuscitation. Biochem. Biophys. Res. Commun. 523: 281-286.

47. Wang M, Fang K, Hong SMC, Kim I, Jang IS, Hong SH. 2018. Medium chain unsaturated fatty acid ethyl esters inhibit persister formation of Escherichia coli via antitoxin HipB. Appl. Microbiol. Biotechnol. 102: 8511-8524.

48. Wood TK, Song S. 2020. Forming and waking dormant cells: the ppGpp ribosome dimerization persister model. Biofilm 2: 100018.

49. Yang H-T, Chen J-W, Rathod J, Jiang Y-Z, Tsai P-J, Hung Y-P, et al. 2017. Lauric acid is an inhibitor of Clostridium difficile growth in vitro and reduces inflammation in a mouse infection model. Front. Microbiol. 8: 2635 .

50. Yang QE, Walsh TR. 2017. Toxin-antitoxin systems and their role in disseminating and maintaining antimicrobial resistance. FEMS Microbiol. Rev. 41: 343-353.

51. Yoon BK, Jackman JA, Valle-González ER, Cho NJ. 2018. Antibacterial free fatty acids and monoglycerides: biological activities, experimental testing, and therapeutic applications. Int. J. Mol. Sci. 19: 1114.

52. Zhou J, Velliou E, Hong SH. 2020. Investigating the effects of nisin and free fatty acid combined treatment on Listeria monocytogenes inactivation. LWT 133: 110115 